\title{
Shock identification and classification in 2D magnetohydrodynamiccompressible turbulence- Orszag-Tang vortex
}

\author{
Ben Snow $^{1, *}$ (D) Andrew Hillier ${ }^{1}$, Giulia Murtas ${ }^{1}$ and Gert J. J. Botha ${ }^{2}$ \\ ${ }^{1}$ Centre for Geophysical and Astrophysical Fluid Dynamics, University of Exeter, Exeter, United Kingdom, and ${ }^{2}$ Department of \\ Mathematics, Physics and Electrical Engineering, Northumbria University, Newcastle upon Tyne, United Kingdom \\ *Corresponding author. Email: b.snow@exeter.ac.uk
}

(Received 12 August 2021; Revised 28 October 2021; Accepted 02 November 2021)

\begin{abstract}
Compressible magnetohydrodynamic (MHD) turbulence is a common feature of astrophysical systems such as the solar atmosphere and interstellar medium. Such systems are rife with shock waves that can redistribute and dissipate energy. For an MHD system, three broad categories of shocks exist (slow, fast, and intermediate); however, the occurrence rates of each shock type are not known for turbulent systems. Here, we present a method for detecting and classifying the full range of MHD shocks applied to the Orszag-Tang vortex. Our results show that the system is dominated by fast and slow shocks, with far less-frequent intermediate shocks appearing most readily near magnetic reconnection sites. We present a potential mechanism that could lead to the formation of intermediate shocks in MHD systems, and study the coherency and abundances of shocks in compressible MHD turbulence.
\end{abstract}

Key words: magnetohydrodynamics; shocks; turbulence

\section{Introduction}

Many astrophysical systems are both highly compressible and turbulent, for example, the solar atmosphere (Carlsson \& Stein, 1992; Houston et al., 2020; Reardon et al., 2008; Ulmschneider, 1970) and interstellar medium (Draine et al., 1983; Elmegreen \& Scalo, 2004). A common feature of compressible turbulence is shocks, where the fluid properties change drastically over a small area. Such sharp structures are important for dissipation. For systems that are governed by magnetohydrodynamic (MHD) equations, there are three broad categories of stable shocks: slow, fast, and intermediate (e.g., Tidman \& Krall, 1971). Understanding the types of shocks that form in a system and where they are likely to form is critical to understand the energy transfer in a turbulent system.

Significant work has been performed to analyze the fast and slow shocks in turbulent systems (e.g., Komissarov, 2012; Orta et al., 2003; Park \& Ryu, 2019); however, intermediate shocks are far less studied, and their existence has been somewhat controversial in the past (Karimabadi, 1995; Wu, 1988). These shock jumps are fully permitted by MHD equations (Hau \& Sonnerup, 1989) and have recently been observed in the solar chromosphere (Houston et al., 2020). However, when and how intermediate shocks can form, and their relative stability, is far from understood. The "strong" intermediate shock (transition from superAlfvénic to subslow) is smoothly connected to the slow-mode shocks (Hau \& Sonnerup, 1989). In two-fluid simulations, it was shown that a slight upstream acceleration of the plasma can result in an intermediate

\footnotetext{
(c) The Author(s), 2021. Published by Cambridge University Press. This is an Open Access article, distributed under the terms of the Creative Commons Attribution licence (http://creativecommons.org/licenses/by/4.0), which permits unrestricted re-use, distribution and reproduction, provided the original article is properly cited.
} 
shock forming (Snow \& Hillier, 2019). Previous studies have suggested that intermediate shocks may be related to magnetic reconnection (La Belle-Hamer et al., 1994; Ugai \& Shimizu, 1994).

Here, we present a method to detect and classify the full range of MHD shocks from a 2D simulation and apply this to the Orszag-Tang (OT) vortex. The results indicate that the vast majority of the shocks are either fast or slow, and hence these shocks are responsible for the majority of the dynamic consequences. Less-frequent intermediate shocks are detected and appear to form in high-current regions, that may arise due to turbulent inflow to a reconnecting region.

\section{Methods}

\subsection{Classification of shocks}

Shocks can be classified based on their upstream and downstream velocity relative to the characteristic speeds of the system (see Table 1). For correct classification, the velocity must be in the shock frame, that is, a frame of reference where the shock is stationary, for example, the de Hoffmann-Teller frame (de Hoffmann \& Teller, 1950), where the electric field is zero either side of the shock. In the de Hoffmann-Teller frame, it is possible to derive an equation that gives all possible steady-state transitions as a function of the upstream (u) and downstream (d) Alfvén Mach numbers, and the upstream plasma- $\beta$ and angle of magnetic field (Hau \& Sonnerup, 1989).

\subsection{Identification technique}

To automatically identify shocks in our simulations, we use the following procedure:

1. Identify shock candidates based on the divergence of the velocity field $(\nabla \cdot \mathbf{v}<0$ is a necessary condition for a shock).

2. Calculate the parallel and perpendicular unit vectors based on the maximum density gradient. The list of possible shocks is then filtered by checking the density gradient along a line perpendicular to the shock front. If the shock candidate is not a local maxima or minima of the perpendicular density gradient, then it is not the center of the feature and is discarded. This prevents the numerical (and physical) finite width of the shock resulting in multiple detections.

3. Estimate the shock frame from the steady-state conservation of mass equation. In the de HoffmanTeller shock frame, the conservation of mass equation becomes $\left[\rho v_{\perp}\right]_{d}^{u}=0$. To transfer the simulation laboratory frame to the shock frame, we need the shock velocity $v_{s}$. By rewriting the mass conservation including this constant shock velocity as $\left[\rho\left(v_{\perp}+v_{s}\right)\right]_{d}^{u}=0$, where $v_{\perp}$ is directly from the simulation, we can estimate the shock velocity by rearranging as $v_{s}=\frac{\rho^{d} v_{\perp}^{d}-\rho^{u} v_{\perp}^{u}}{\rho^{u}-\rho^{d}}$.

4. Compare the upstream and downstream Mach numbers to calculate the shock transition.

Table 1. Possible stable magnetohydrodynamic shock transitions based on the upstream and downstream states

\begin{tabular}{lll}
\hline Upstream state $(u)$ & Downstream state $(d)$ & Shock classification \\
\hline Superfast (1) & Subfast (2) & $1 \rightarrow 2$ Fast \\
\hline Superslow (3) & Subslow (4) & $3 \rightarrow 4$ Slow \\
\hline Superfast (1) & Superslow (3) & $1 \rightarrow 3$ Intermediate \\
\hline Superfast (1) & Subslow (4) & $1 \rightarrow 4$ Intermediate \\
\hline Subfast (2) & Superslow (3) & $2 \rightarrow 3$ Intermediate \\
\hline Subfast (2) & Subslow (4) & $2 \rightarrow 4$ Intermediate \\
\hline
\end{tabular}

Note: Transitions of the form $u>d$ are entropy-forbidden, so not listed here. 
There are a number of assumptions that go into this identification technique, with the strongest being that the shocks are steady-state. In a highly dynamic simulation, this may prevent transient shocks being detected as readily and could lead to erroneous shock detection. As such, our identification procedure is likely an underestimate of the number of shocks in the system. Furthermore, we include additional checks to confirm the compressibility is greater than unity for all shocks, and that there is a reversal in the magnetic field across the interface for the intermediate shocks.

\subsection{MHD simulation}

To test this shock detection code, we use the OT shock vortex (Orszag \& Tang, 1979). The OT initial conditions produce a decaying compressible turbulent system starting from the following initial conditions:

$$
\begin{array}{lll}
v_{x}=-v_{0} \sin (2 \pi y), & (1) & \rho=25 /(36 \pi), \\
v_{y}=v_{0} \sin (2 \pi x), & (2) & P=5 /(12 \pi), \\
B_{x}=-B_{0} \sin (2 \pi y), & (3) & v_{0}=1, \\
B_{y}=B_{0} \sin (4 \pi y), & (4) & B_{0}=1 / \sqrt{4 \pi}
\end{array}
$$

for density $\rho$, pressure $P$, velocity $\mathbf{v}=\left[v_{x}, v_{y}, 0\right]$, and magnetic field $\mathbf{B}=\left[B_{x}, B_{y}, 0\right]$. The OT vortex has been well studied in the literature (Dahlburg \& Picone, 1989; Jiang \& Wu, 1999; Parashar et al., 2009; Picone \& Dahlburg, 1991; Uritsky et al., 2010). The initial conditions are evolved in 2D for ideal MHD equations using the fourth-order central-difference solver in the (PIP) code (Hillier et al., 2016), which has been used previously to study shocks (e.g., Snow \& Hillier, 2021). The simulations are performed using $1,024 \times 1,024$ cells with periodic boundary conditions.

\section{Results}

Figure 1 shows the evolution of the OT vortex through time, with the identified shock pixels colored according to their classification. The simulation shows the full range of shock transitions exists in our simulation, with the most prevalent being the fast- and slow-mode shocks. Intermediate shocks are detected in the simulation; however, these are far less-frequent and appear later than the fast or slow shocks.

Figure 1e shows the number and type of detected shocks in the simulation through time. At very early times in the simulation $(t<0.15)$, the detections of shocks are sporadic, because the system is evolving rapidly and the assumption of a steady-state shock is not valid. After $t=0.15$, large-scale fast-mode shocks are generated due to the initial conditions of the OT vortex. As the system develops and the shocks interact, slow-mode shocks become increasingly detected in the system, and fast and slow shocks have comparable detection counts near the end of the simulation $(t=1)$. The relative counts of fast- and slowmode shocks are likely dependent on the initial conditions of the system (Dahlburg \& Picone, 1989; Picone \& Dahlburg, 1991).

A metric to determine the coherency of shocks can be calculated by measuring the number of similar shocks within a given radius. Here, we use a radius of three grid cells and classify any shocks that have more than six nearby shocks of the same type as coherent. Figure $1 \mathrm{f}$ shows the proportion of coherent fast and slow shocks through time. At early times in the simulation, the system is dominated by long, continuous, fast-mode shocks, and hence the proportion of coherent shocks is very high. Because the simulation evolves and the fast-mode shocks interact and break up, the coherency decreases. For the slow-mode shocks, the coherency is fairly constant through time at around $40 \%$. From Figure $1 \mathrm{~b}-\mathrm{d}$, one can see that the large-scale slow-mode shocks may exist, but are not detected as continuous features, possibly due to either limitations of the detection method. 

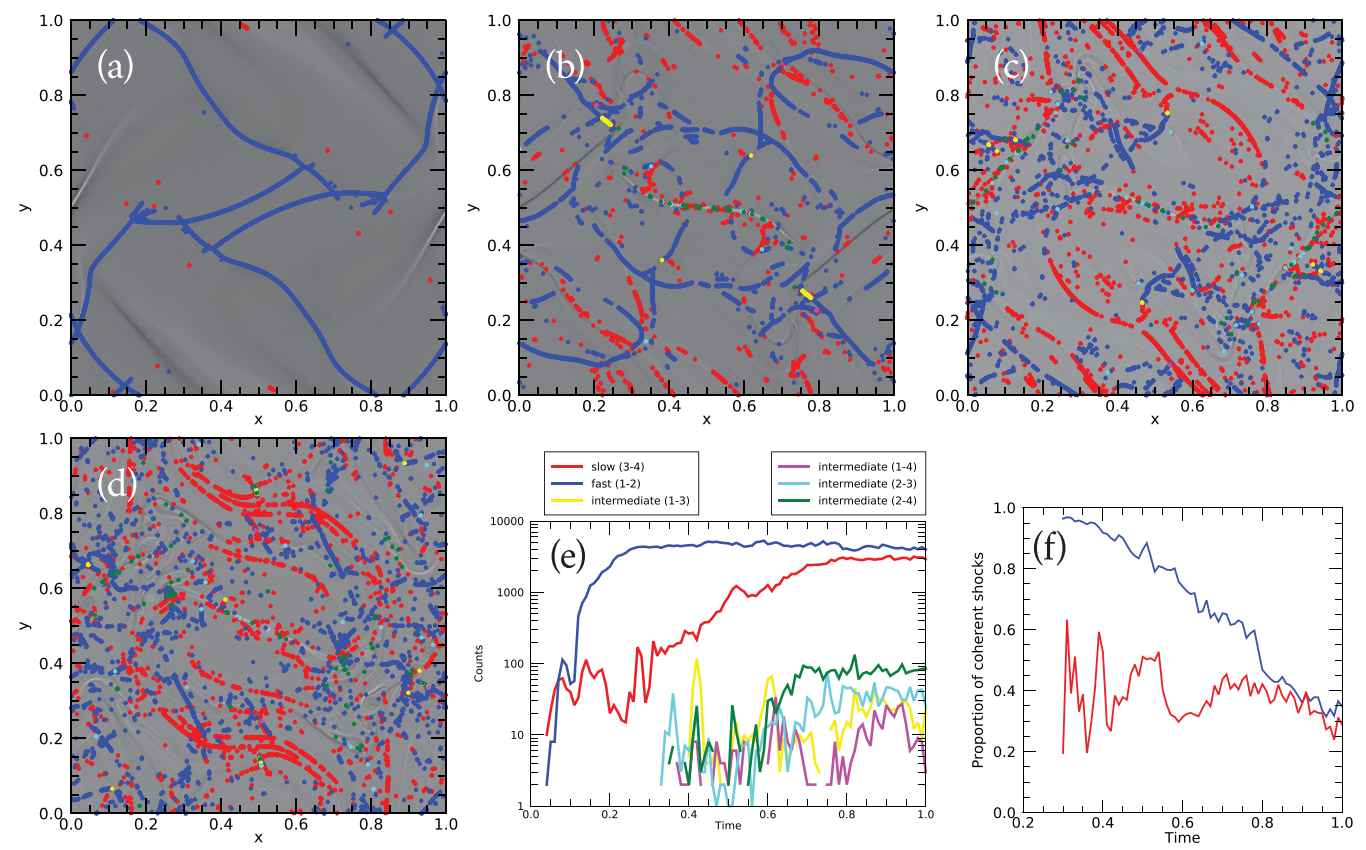

Figure 1. Shocks detected in the Orszag-Tang vortex in magnetohydrodynamic at $t=0.3$ (a), 0.6 (b), 0.8 (c), and 1.0 (d). Background shows the out-of-plane current. Overplotted are the detected slow (red), fast (blue), and intermediate (yellow, magenta, cyan, and green) shocks. The occurrence counts of each type of shock through time are shown in the panel (e). The panel (f) shows the coherency of fast and slow shocks through time.

It has been suggested that the corrugation instability (e.g., Stone \& Edelman, 1995) may result in far fewer slow-mode shocks being detectable in turbulent simulations (Park \& Ryu, 2019). However, here, there are comparable numbers of fast and slow shocks in the system after $t=0.8$, and large un-corrugated coherent slow-mode shock structures exist in the simulation (see Figure $1 \mathrm{~b}-\mathrm{d}$ ). A recent study has shown that the corrugation instability can actually increase the number of detected shock pixels due to an increased shock length (Snow \& Hillier, 2021).

\subsection{Potential mechanism for the formation of intermediate shocks}

The intermediate shocks appear to form near and around high-current regions. As such, one may consider the link between magnetic reconnection and intermediate shocks in turbulent systems. Note that not all high current regions feature intermediate shocks, but intermediate shocks are usually located at a high-current region. For fast magnetic reconnection, one may expect switch-off slow-mode shocks (e.g., Petschek, 1964), where the inflow Alfvén Mach number is unity. High-resolution MHD simulations have shown that switch-off slow-mode shocks are integral to plasmoid formation (Zenitani \& Miyoshi, 2011). Now, since a switch-off slow-mode shock has an inflow Alfvén Mach number of 1 , it is on the cusp of being a $2 \rightarrow 4$ intermediate shock (where the inflow is super-Alfvàic and the downstream is subslow, e.g., Figure 1 in Hau \& Sonnerup, 1989). As such, for a turbulent reconnection region, one would expect variations in the inflow quantities that could result in a super-Alfvénic inflow and an intermediate shock. This appears to be the formation mechanism occurring here. Specifically isolating a plasmoid in a highresolution MHD simulation, one sees remarkable similarities to the plasmoid schematic in Zenitani and Miyoshi (2011); however, here, intermediate shocks form on the inflow (see Figure 2). In this example figure, the inflow to the plasmoid is asymmetric, and slow-mode shocks form on the lower inflow, whereas intermediate shocks form on the upper inflow. 


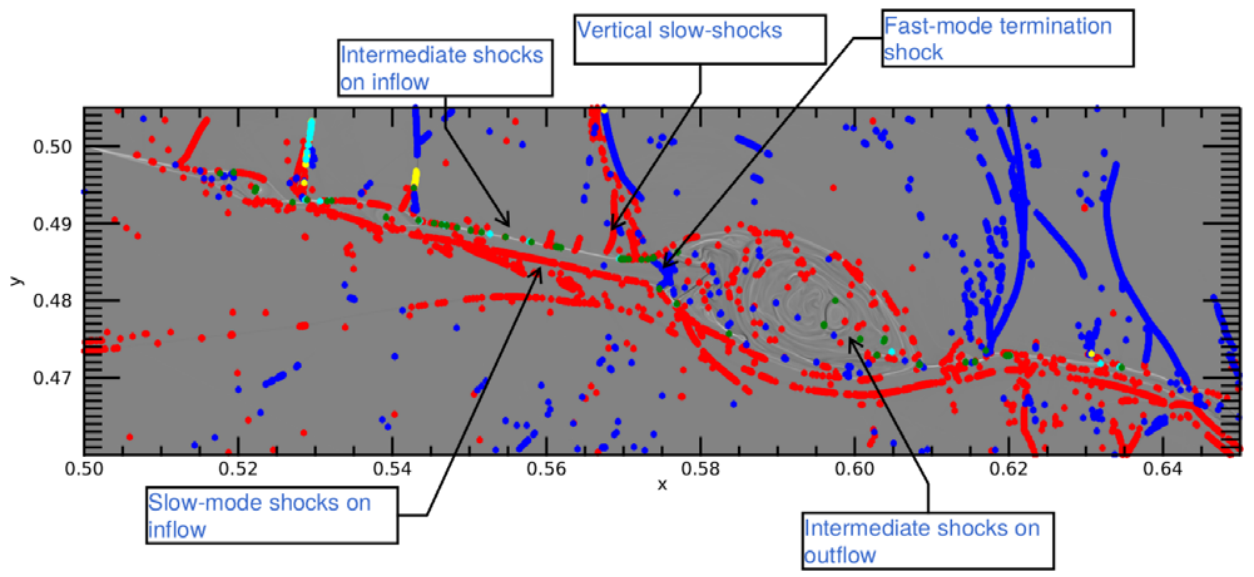

Figure 2. Plasmoid with intermediate shocks forming on inflow region. Fundamental structures of the plasmoid are annotated.

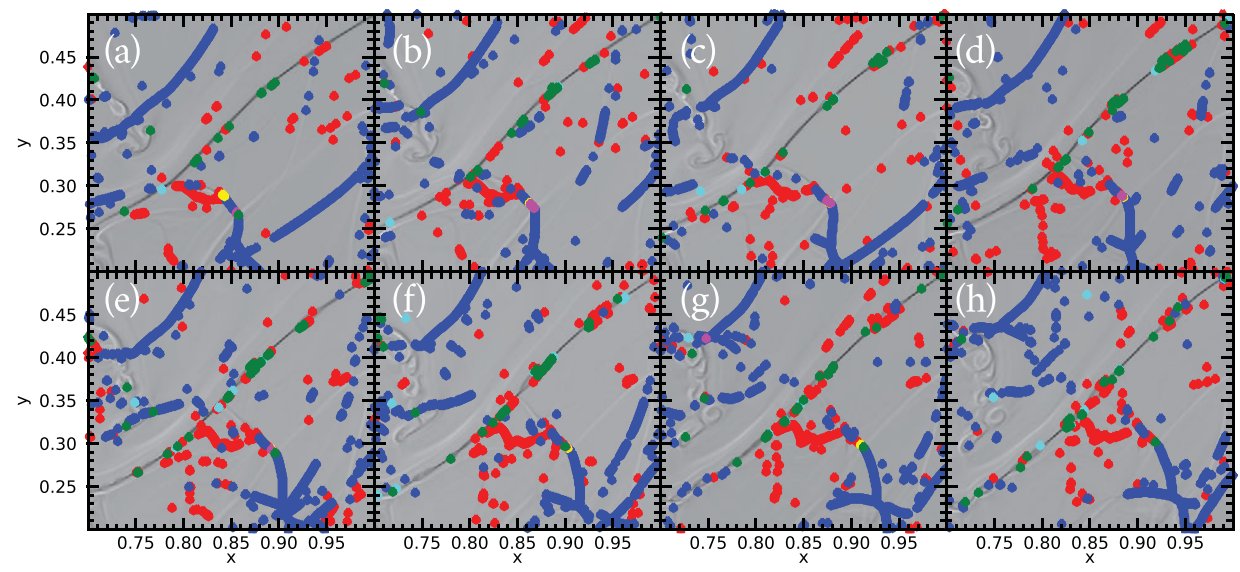

Figure 3. Snapshots of a reconnecting region in the time frame $t=0.66-0.73$ (from [a]-[h]) at intervals of 0.01 . Background color is the out-of-plane current density.

In terms of relative stability, in these simulations, the intermediate shocks can be regularly detected across a few time steps, and by eye, one can track certain shocks across outputs; however, others are less stable (see Figure 3). Through time, the intermediate shocks are routinely detected at the high-current regions. Further work is needed to analyze the stability and evolution of intermediate shocks.

\subsection{Consistency of results at higher grid resolutions}

A high-resolution $\left(16,384^{2}\right)$ simulation of the OT vortex was performed to analyze the consistency of the results. At time $t=1$, the number of pixels that satisfy a shock jump condition in the high-resolution simulation are: 160,819 (slow), 316,029 (fast), 350 (intermediate 1-2), 23 (intermediate 1-4), 836 (intermediate 2-3), and 1,394 (intermediate 2-4). The increased grid resolution has led to many more shocks being detected; however, proportionally, the results are similar. There is a factor of $\approx 2$ faster than slow shocks (as in the $1,024^{2}$ simulation; see Figure 1). Intermediate shocks are proportionally less-frequent; intermediate $2-4$ shocks are $\approx 3 \%$ as frequent as slow shocks at $1,024^{2}$, and $\approx 0.1 \%$ in the high-resolution simulation. This may be due to the larger scale shocks contributing more to the overall shock count and 
requires further study. However, fast and slow shocks are most abundant, and the intermediate shocks form at high-current regions in both simulations indicating that these are consistent results.

\section{Conclusion}

The developed method for identifying shocks in MHD systems detects the full range of MHD shocks in the OT vortex. The MHD simulation is dominated by fast and slow shocks implying that the vast majority of the shock-related dissipation occurs due to fast and slow shocks. Furthermore, the fast and slow shocks occur with roughly equal detected pixel counts at $t=1$ implying that the corrugation instability is not preventing slow-mode shocks from being detected. Less-frequent intermediate transitions are also detected in the simulation that appear to be connected to high-current regions. In particular, the 2-4 intermediate shock may be formed due to fluctuations of the inflow velocity from a reconnectiongenerated switch-off shock. Further work is needed to analyze the evolution and stability of intermediate shocks occurring in turbulent MHD systems.

Acknowledgments. We would like to thank the stimulating discussions with Joanne Mason, Pierre Lesaffre, Andrew Lehmann, and Thibaud Richard.

Funding Statement. B.S. and A.H. are supported by STFC research grant ST/R000891/1 and ST/V000659/1.

Conflict of Interest. The authors have no conflicts of interest to declare.

Authorship Contributions. B.S. designed the study and wrote the manuscript. A.H. and G.J.J.B. provided insight and assisted in understanding the results. G.M. assisted in benchmarking the shock identification code.

Data availability Statement. The simulation data from this study are available from B.S. upon reasonable request. The (PIP) code is available at https://github.com/AstroSnow/PIP. The IDL scripts used for this manuscript are available at https://github. com/AstroSnow/Orszag-Tang-Shock-Detection.

\section{References}

Carlsson, M., \& Stein, R. F. (1992). Non-LTE radiating acoustic shocks and CA II K2V bright points. The Astrophysical Journal, 397, L59.

Dahlburg, R. B., \& Picone, J. M. (1989). Evolution of the Orszag-Tang vortex system in a compressible medium. I. Initial average subsonic flow. Physics of Fluids B: Plasma Physics, 1, 2153-2171.

de Hoffmann, F., \& Teller, E. (1950). Magneto-hydrodynamic shocks. Physical Review, 80, 692-703.

Draine, B. T., Roberge, W. G., \& Dalgarno, A. (1983). Magnetohydrodynamic shock waves in molecular clouds. The Astrophysical Journal, 264, 485-507.

Elmegreen, B. G., \& Scalo, J. (2004). Interstellar turbulence I: Observations and processes. Annual Review of Astronomy and Astrophysics, 42, 211-273.

Hau, L.-N., \& Sonnerup, B. U. O. (1989). On the structure of resistive MHD intermediate shocks. Journal of Geophysical Research, 94, 6539-6551.

Hillier, A., Takasao, S., \& Nakamura, N. (2016). The formation and evolution of reconnection-driven, slow-mode shocks in a partially ionised plasma. Astronomy \& Astrophysics, 591, A112.

Houston, S. J., Jess, D. B., Keppens, R., Stangalini, M., Keys, P. H., Grant, S. D. T., Jafarzadeh, S., McFetridge, L. M., Murabito, M., Ermolli, I., \& Giorgi, F. (2020). Magnetohydrodynamic nonlinearities in sunspot atmospheres: Chromospheric detections of intermediate shocks. The Astrophysical Journal, 892, 49.

Jiang, G.-S., \& Wu, C.-C. (1999). A high-order WENO finite difference scheme for the equations of ideal magnetohydrodynamics. Journal of Computational Physics, 150, 561-594.

Karimabadi, H. (1995). Physics of intermediate shocks: A review. Advances in Space Research, 15, 507-520.

Komissarov, S. S. (2012). Shock dissipation in magnetically dominated impulsive flows. Monthly Notices of the Royal Astronomical Society, 422, 326-346.

La Belle-Hamer, A. L., Otto, A., \& Lee, L. C. (1994). Magnetic reconnection in the presence of sheared plasma flow: Intermediate shock formation. Physics of Plasmas, 1, 706-713.

Orszag, S. A., \& Tang, C. M. (1979). Small-scale structure of two-dimensional magnetohydrodynamic turbulence. Journal of Fluid Mechanics, 90, 129-143. 
Orta, J. A., Huerta, M. A., \& Boynton, G. C. (2003). Magnetohydrodynamic shock heating of the solar corona. The Astrophysical Journal, 596, 646-655.

Parashar, T. N., Shay, M. A., Cassak, P. A., \& Matthaeus, W. H. (2009). Kinetic dissipation and anisotropic heating in a turbulent collisionless plasma. Physics of Plasmas, 16, 032310.

Park, J., \& Ryu, D. (2019). Shock waves and energy dissipation in magnetohydrodynamic turbulence. The Astrophysical Journal, 875, 2.

Petschek, H. E. (1964). Magnetic field annihilation (Vol. 50, p. 425). NASA Special Publications.

Picone, J. M., \& Dahlburg, R. B. (1991). Evolution of the Orszag-Tang vortex system in a compressible medium. II. Supersonic flow. Physics of Fluids B, 3, 29-44.

Reardon, K. P., Lepreti, F., Carbone, V., \& Vecchio, A. (2008). Evidence of shock-driven turbulence in the solar chromosphere. The Astrophysical Journal, 683, L207.

Snow, B., \& Hillier, A. (2019). Intermediate shock sub-structures within a slow-mode shock occurring in partially ionised plasma. Astronomy \& Astrophysics, 626, A46.

Snow, B., \& Hillier, A. (2021). Stability of two-fluid partially ionized slow-mode shock fronts. Monthly Notices of the Royal Astronomical Society, 506, 1334-1345.

Stone, J. M., \& Edelman, M. (1995). The corrugation instability in slow magnetosonic shock waves. The Astrophysical Journal, 454, 182.

Tidman, D. A., \& Krall, N. A. (1971). Shock waves in collisionless plasmas. Wiley.

Ugai, M., \& Shimizu, T. (1994). Computer studies on noncoplanar slow and intermediate shocks associated with the sheared fast reconnection mechanism. Physics of Plasmas, 1, 296-307.

Ulmschneider, P. (1970). On frequency and strength of shock waves in the solar atmosphere. Solar Physics, 12, $403-415$.

Uritsky, V. M., Pouquet, A., Rosenberg, D., Mininni, P. D., \& Donovan, E. F. (2010). Structures in magnetohydrodynamic turbulence: Detection and scaling, 82, 056326.

Wu, C. C. (1988). The MHD intermediate shock interaction with an intermediate wave: Are intermediate shocks physical?. Journal of Geophysical Research: Space Physics, 93, 987-990.

Zenitani, S., \& Miyoshi, T. (2011). Magnetohydrodynamic structure of a plasmoid in fast reconnection in low-beta plasmas. Physics of Plasmas, 18, 022105.

Cite this article: Snow B, Hillier A, Murtas G, Botha GJJ (2021). Shock identification and classification in 2D magnetohydrodynamiccompressible turbulence-Orszag-Tang vortex Experimental Results, 2, e35, 1-10. https://doi.org/ $10.1017 /$ exp.2021.28 


\title{
Peer Reviews
}

\author{
Reviewing editor: Prof. Stefano Camera ${ }^{1,2}$ \\ ${ }^{1}$ Universita degli Studi di Torino, Physics, Via Pietro Giuria, 1, Torino, Italy, 10124 \\ ${ }^{2}$ University of the Western Cape, Physics \& Astronomy, Bellville, South Africa, 7535
}

This article has been accepted because it is deemed to be scientifically sound, has the correct controls, has appropriate methodology and is statistically valid, and has been sent for additional statistical evaluation and met required revisions.

doi:10.1017/exp.2021.28.pr1

\section{Review 1: Shock identification and classification in 2D MHD compressible turbulence - Orszag- Tang vortex}

Reviewer: Dr. Philip Mocz (D)

Lawrence Livermore National Laboratory, Livermore, California, United States, 94550-5507

Date of review: 14 September 2021

(C) The Author(s), 2021. Published by Cambridge University Press. This is an Open Access article, distributed under the terms of the Creative Commons Attribution licence (http://creativecommons.org/licenses/by/4.0), which permits unrestricted re-use, distribution and reproduction, provided the original article is properly cited.

Conflict of interest statement. Reviewer declares none

Comments to the Author: This is an excellent paper that characterizes fast, slow, and intermediate shocks in the Orszag-Tang vortex problem -- a classic test problem for numerical simulations and an example of supersonic decaying turbulence. I recommend it for publication, following consideration of my comments below.

I found the presentation very clear and the analysis straightforward. The results about which types of shocks are responsible for dissipating energy in this test problem is interesting.

The one comment I have is that the authors' simulate the ideal MHD equations at a single fixed resolution. Without physical resistivity, the solution is known not to converge, and dissipation and formation of plasmoids is resolution dependent (e.g. one would not even form plasmoids at lowresolution $64 \times 64$ ). So my question is, how robust are the findings of which shock types dominate to increased resolution, e.g. $2048^{\wedge} 2,4096^{\wedge} 2,8192^{\wedge} 2$ ? My experience simulating the OT problem is that plasmoid structure can change significantly with increased resolution. It is possible that results/dissipation are converged in $2 \mathrm{D}$ because $2 \mathrm{D}$ turbulence is an inverse-cascade. Numerical studies of dissipation in $3 \mathrm{D}$ problems though, where you have cascade to small-scales, have proved to be more difficult due to effects of numerical viscosity/resistivity.

Typos:

* page 4. "vortex.As" --> "vortex. As"

\section{Score Card}

Presentation

Is the article written in clear and proper English? (30\%)

Is the data presented in the most useful manner? (40\%)

Does the paper cite relevant and related articles appropriately? (30\%) 
Does the abstract correctly embody the content of the article? (25\%)

Does the introduction give appropriate context? (25\%)

Is the objective of the experiment clearly defined? (25\%)

Analysis

Does the discussion adequately interpret the results presented? (40\%)

Is the conclusion consistent with the results and discussion? (40\%)

Are the limitations of the experiment as well as the contributions of the experiment clearly outlined? $(20 \%)$ 


\section{Review 2: Shock identification and classification in 2D MHD compressible turbulence - Orszag- Tang vortex}

Reviewer: Dr. Reinaldo Santos de Lima iD

University of Sao Paulo, stronomy, Rua do Matao 1226, Sao Paulo, Sao Paulo, Brazil, 05508-900

Date of review: 27 October 2021

(C) The Author(s), 2021. Published by Cambridge University Press. This is an Open Access article, distributed under the terms of the Creative Commons Attribution licence (http://creativecommons.org/licenses/by/4.0), which permits unrestricted re-use, distribution and reproduction, provided the original article is properly cited.

Conflict of interest statement. Reviewer declares none.

Comments to the Author: Both the methods and the resolution employed in the numerical calculations can be critical to the statistical results presented in this study. I recommend the authors to report the sensitivity of their results with respect to the resolution (convergence test), and to the Riemann solver used.

\section{Score Card}

Presentation

Is the article written in clear and proper English? (30\%)

Is the data presented in the most useful manner? (40\%)

Does the paper cite relevant and related articles appropriately? (30\%)

Context

Does the abstract correctly embody the content of the article? (25\%)

Does the introduction give appropriate context? (25\%)

Is the objective of the experiment clearly defined? (25\%)

Analysis

Are the limitations of the experiment as well as the contributions of the experiment clearly outlined? (20\%) 